\title{
Neonatal sepsis definitions from randomised clinical trials
}

Rían Hayes ${ }^{1}$, Jack Hartnett ${ }^{1}$, Gergana Semova ${ }^{1}$, Cian Murray ${ }^{1}$, Katherine Murphy ${ }^{1}$, Leah Carroll ${ }^{1}$, Helena Plapp ${ }^{1}$, Louise Hession ${ }^{1}$, Jonathan O'Toole ${ }^{1}$, Danielle McCollum ${ }^{1}$, Edna Roche ${ }^{1}$, Elinor Jenkins ${ }^{1}$, David Mockler ${ }^{2}$, Tim Hurley ${ }^{1,3}$, Matthew McGovern ${ }^{1,3}$, John Allen ${ }^{1,3,4}$, Judith Meehan ${ }^{1,4}$, Frans B. Plötz ${ }^{5,6}$, Tobias Strunk ${ }^{7,8}$, Willem P. de Boode ${ }^{9}$, Richard Polin ${ }^{10}$, James L. Wynn ${ }^{11,12}$, Marina Degtyareva ${ }^{13}$, Helmut Küster ${ }^{14}$, Jan Janota ${ }^{15,16}$, Eric Giannoni ${ }^{17}$, Luregn J. Schlapbach ${ }^{18,19,20}$, Fleur M. Keij ${ }^{21}$, Irwin K. M. Reiss ${ }^{21}$, Joseph Bliss 22 , Joyce M. Koenig ${ }^{23}$, Mark A. Turner ${ }^{24}$, Christopher Gale ${ }^{25}$, Eleanor J. Molloy 1,3,4,26,27 and On behalf of the Infection, Inflammation, Immunology and Immunisation (I4) section of the European Society for Paediatric Research (ESPR)

(c) The Author(s) 2021

INTRODUCTION: Neonatal sepsis is a leading cause of infant mortality worldwide with non-specific and varied presentation. We aimed to catalogue the current definitions of neonatal sepsis in published randomised controlled trials (RCTs).

METHOD: A systematic search of the Embase and Cochrane databases was performed for RCTs which explicitly stated a definition for neonatal sepsis. Definitions were sub-divided into five primary criteria for infection (culture, laboratory findings, clinical signs, radiological evidence and risk factors) and stratified by qualifiers (early/late-onset and likelihood of sepsis).

RESULTS: Of 668 papers screened, 80 RCTs were included and 128 individual definitions identified. The single most common definition was neonatal sepsis defined by blood culture alone $(n=35)$, followed by culture and clinical signs $(n=29)$, and then laboratory tests/clinical signs $(n=25)$. Blood culture featured in 83 definitions, laboratory testing featured in 48 definitions while clinical signs and radiology featured in 80 and 8 definitions, respectively.

DISCUSSION: A diverse range of definitions of neonatal sepsis are used and based on microbiological culture, laboratory tests and clinical signs in contrast to adult and paediatric sepsis which use organ dysfunction. An international consensus-based definition of neonatal sepsis could allow meta-analysis and translate results to improve outcomes.

Pediatric Research; https://doi.org/10.1038/s41390-021-01749-3

\section{INTRODUCTION}

An estimated 3,000,000 newborns are affected by sepsis annually ${ }^{1}$ with mortality set to reach 375,000 in 2019 (ref. ${ }^{2}$ ). Despite ongoing advances in molecular diagnostics, ${ }^{3}$ the accurate and timely diagnosis of sepsis in neonates remains challenging. Current conventional gold standard diagnosis of sepsis based on microbial culture does not appear to reliably rule-out sepsis, with reported rates of 'culture-negative' or 'suspected' sepsis varying widely in the literature. While some experts advocate to consider sepsis evaluations completed after $48-72 \mathrm{~h}$ of negative blood cultures ${ }^{4,5}$ data available from two large randomised controlled trials (RCTs) in recent years (INIS ${ }^{6}$ and $\mathrm{ELFIN}^{7}$ ) show culture-negative sepsis rates of $56 \%$ and $46 \%$, respectively.

Antibiotic overuse and resistance are increasingly common but under-recognition of sepsis remains an issue resulting in campaigns from the World Health Organisation (WHO). ${ }^{8}$

Sepsis-3 (ref. ${ }^{9}$ ), the recent consensus definitions for sepsis and septic shock in adults, primarily uses multiorgan dysfunction rather than microbial culture in the screening and diagnosis of sepsis. Even when microbiological tests are completed, culturepositive 'sepsis' is observed in only $30-40 \%$ of cases in adults. Neonatal sepsis literature is therefore at variance with adult and paediatric sepsis consensus and a greater emphasis on multiorgan

\footnotetext{
'Discipline of Paediatrics, Trinity College Dublin, the University of Dublin \& Children's Hospital Ireland (CHI) at Tallaght, Dublin, Ireland. ${ }^{2}$ John Stearne Medical Library, Trinity College Dublin, St. James' Hospital, Dublin, Ireland. ${ }^{3}$ Trinity Translational Medicine Institute, St James Hospital, Dublin, Ireland. ${ }^{4}$ Trinity Research in Childhood Centre (TRiCC), Trinity College Dublin, Dublin, Ireland. ${ }^{5}$ Department of Paediatrics, Tergooi Hospital, Blaricum, The Netherlands. ${ }^{6}$ Department of Paediatrics, Amsterdam UMC, University of Amsterdam, Emma Children's Hospital, Amsterdam, The Netherlands. ${ }^{7}$ Neonatal Health and Development, Telethon Kids Institute, Perth, WA, Australia. ${ }^{8}$ Neonatal Directorate, King Edward Memorial Hospital for Women, Perth, WA, Australia. ${ }^{9}$ Radboud Institute for Health Sciences, Department of Neonatology, Radboud University Medical Center, Amalia Children's Hospital, Nijmegen, The Netherlands. ${ }^{10}$ Division of Neonatal-Perinatal Medicine, Department of Pediatrics, Columbia University Medical Center, New York City, NY, USA. ${ }^{11}$ Department of Pediatrics, University of Florida, Gainesville, FL, USA. ${ }^{12}$ Department of Pathology, Immunology, and Laboratory Medicine, University of Florida, Gainesville, FL, USA. ${ }^{13}$ Department of Neonatology, Pirogov Russian National Research Medical University, Moscow, Russia. ${ }^{14}$ Neonatology, Clinic for Paediatric Cardiology, Intensive Care and Neonatology, University Medical Centre Göttingen, Göttingen, Germany. ${ }^{15}$ Neonatal Unit, Department of Obstetrics and Gynecology, Motol University Hospital and Second Faculty of Medicine, Prague, Czech Republic. ${ }^{16}$ Institute of Pathological Physiology, First Faculty of Medicine, Charles University, Prague, Czech Republic. ${ }^{17}$ Clinic of Neonatology, Department Mother-Woman-Child, Lausanne University Hospital and University of Lausanne, Lausanne, Switzerland. ${ }^{18}$ Paediatric Critical Care Research Group, Child Health Research Centre, University of Queensland, Brisbane, Australia. ${ }^{19}$ Paediatric Intensive Care Unit, Queensland Children's Hospital, Brisbane, Australia. ${ }^{20}$ Department of Pediatrics, Bern University Hospital, University of Bern, Bern, Switzerland. ${ }^{21}$ Department of Pediatrics, Division of Neonatology, Erasmus MC-Sophia Children's Hospital, Rotterdam, The Netherlands. ${ }^{22}$ Department of Pediatrics, Women \& Infants Hospital of Rhode Island, Alpert Medical School of Brown University, Providence, USA. ${ }^{23}$ Division of Neonatology, Saint Louis University, Edward Doisy Research Center, St. Louis, MO, USA. ${ }^{24}$ Institute of Translational Medicine, University of Liverpool, Centre for Women's Health Research, Liverpool Women's Hospital, Liverpool, UK. ${ }^{25}$ Neonatal Medicine, School of Public Health, Faculty of Medicine, Chelsea and Westminster campus, Imperial College London, London, UK. ${ }^{26}$ Paediatrics, Coombe Women's and Infant's University Hospital, Dublin, Ireland. ${ }^{27}$ Neonatology, CHI at Crumlin, Dublin, Ireland. ${ }^{\varpi}$ email: Eleanor.molloy@tcd.ie
}

Received: 9 July 2021 Revised: 27 August 2021 Accepted: 31 August 2021

Published online: 06 November 2021 
dysfunction may be merited. The neonatal Sequential Organ Failure Assessment Score (nSOFA) ${ }^{10}$ characterises neonatal organ dysfunction and predicts mortality in this setting, and thus the potential for its use in the definition of neonatal sepsis. Measures of neonatal organ dysfunction have been developed but remain distinct from definitions of infection or sepsis at present.

Neonatal sepsis, especially in vulnerable (e.g. very low birth weight) populations, is strongly associated with increased rates of complications of prematurity ${ }^{11,12}$ and adverse neurodevelopmental outcomes. ${ }^{13-18}$ These unfavourable outcomes emphasise the need for rapid recognition of sepsis and initiation of antibiotic therapy. However, unnecessary empiric antibiotic prescription is associated with increasing long term morbidity and mortality, ${ }^{19-28}$ and current antibiotic prescribing habits have been shown to vary widely internationally. ${ }^{29-32}$ These issues emphasise the need for a consensus definition of neonatal sepsis so that standardised outcomes data can feed back into diagnostic and therapeutic improvement. ${ }^{33}$

In this study we aimed to systematically review the definitions of neonatal sepsis used in published RCTs. This process is in tandem with a separate analysis ${ }^{34}$ which examines the use of definitions of neonatal sepsis in observational and experimental studies from neonatal surveillance and research networks. The goal here is to identify commonalities and differences between definitions of neonatal sepsis in order to define core themes for Delphi and consensus processes to develop a universally accepted definition for neonatal sepsis.

\section{METHODS \\ Search strategy}

In May 2019, the Embase and Cochrane databases were searched with the algorithm ('neonatal' OR 'newborn') AND ('sepsis' OR 'septicaemia'). No date restrictions were imposed on results. Only randomised clinical trials in the English language which explicitly stated a definition for neonatal sepsis were included. All other publications, including observational studies, reviews and RCTs for which a full text was not available, and RCTs that included nonneonatal paediatric patients were excluded.

\section{Data collection and analysis}

The following data were extracted from the included studies: year of publication, sample size, verbatim definition of sepsis, and primary and secondary outcomes measured. After a preliminary review of the data, the authors characterised five primary criteria from which all the definitions of neonatal sepsis were composed: microbiological culture, clinical signs of infection, laboratory signs of infection, radiological signs of infection, and the presence of risk factors for infection. Many RCTs presented algorithmic definitions of neonatal sepsis, e.g. 'Neonatal sepsis was defined as a positive microbial culture plus either laboratory signs of sepsis or clinical signs of sepsis'. To enable adequate analysis, these algorithms were broken down by their component primary criteria. For instance, in the above definition that diagnosed neonatal sepsis as 'positive culture plus clinical signs or laboratory signs of infection' we took this as two separate definitions of neonatal sepsis: (1) culture plus clinical signs of infection and (2) culture plus laboratory signs of infection. For each primary criterion, a detailed breakdown of the relevant secondary criteria was collected. Where definitions included microbiological culture as a criterion, the collected secondary criteria were: culture site(s), the number of samples required to be positive, pathogens cultured for, the time the sample was taken, and the incubation time. For laboratory signs, clinical signs, radiological signs, and risk factors for infection, the frequency of individual signs was recorded. Where definitions specified a required number of positive signs in a specific category, these figures were recorded. Many definitions of neonatal sepsis included qualifiers (e.g. early/ late, suspected/proven, coagulase-negative staphylococci (CoNS) and additional criteria for severe sepsis/septic shock). These qualifiers were recorded and a sub-analysis of the criteria they used was performed.

\section{RESULTS}

\section{Search results}

A total of 668 papers were identified by the search, of which 344 were excluded based on screening of the title and abstract; 80 of the remaining 324 studies met the inclusion criteria for the review as RCTs and were included in the analysis of the full text. The selection of studies was undertaken in accordance with the PRISMA (Preferred Reporting Items for Systematic Reviews and Meta-Analyses) guidelines (Fig. 1).

\section{Study characteristics}

A total of 80 randomised clinical trials ${ }^{4,5,8,35-122}$ were included in the review from 1986 to 2019 yielding a total of 128 individual definitions of neonatal sepsis when broken down by component primary criteria (Table 1): microbiological culture, clinical signs of infection, laboratory signs of infection, radiological signs of infection, and the presence of risk factors for infection. The tabulated raw dataset is available in Appendix 1. The total sample size comprised by the trials was 40,992 neonates, the median sample size was 150 , and the mean sample size was 512 . The most represented primary criterion was microbiological culture, which was a component of 83 separate definitions. The frequency of other primary criteria appeared as follows: clinical signs of infection $(n=81)$, biochemical/haematological signs of infection $(n=48)$, radiological signs of infection $(n=8)$, risk factors for infection $(n=2)$. None of the included definitions were consensus or guideline definitions.

\section{Primary outcomes}

\section{(1) Microbiological culture}

Microbiological culture was a component of 83 out of 128 definitions of neonatal sepsis (Table 1). These 83 definitions were from 68 different papers.

Culture source: Of the 83 culture-related definitions, 74 specified a site the culture sample was taken from, while 9 mentioned 'culture' without specifying a sample site. Of the 74 that specified culture sites, the frequency of each site mentioned is outlined in Table 2. Three definitions required two positive cultures to diagnose sepsis.

Pathogen: 12 definitions specified a pathogen sought by microbiological culture. Five specified bacteria, 4 specified fungi, and the remainder mentioned 'known virulent pathogens', or 'not Staphylococcus epidermidis'.

Incubation time: Only one study mentioned incubation time, which outlined that a microorganism was regarded as infectious if it grew within $48 \mathrm{~h}$ incubation.

(2) Clinical signs of infection

Clinical signs were a component of 81 definitions. Seven defined sepsis based on signs alone and 74 defined sepsis based on signs in conjunction with other primary criteria, culture being the most common. These 81 definitions represent 35 RCTs that gave only 1 definition of sepsis that included clinical signs, and a further 18 papers which had multiple definitions that included clinical signs. Ten of these 18 papers gave a definition based on culture and clinical signs, and another definition based on laboratory criteria and clinical signs. Several other papers which included clinical signs in their definitions also gave multiple definitions that distinguished between possible and probable sepsis. Out of 81 definitions of sepsis to include clinical signs, 41 specified the signs that were looked for (either 


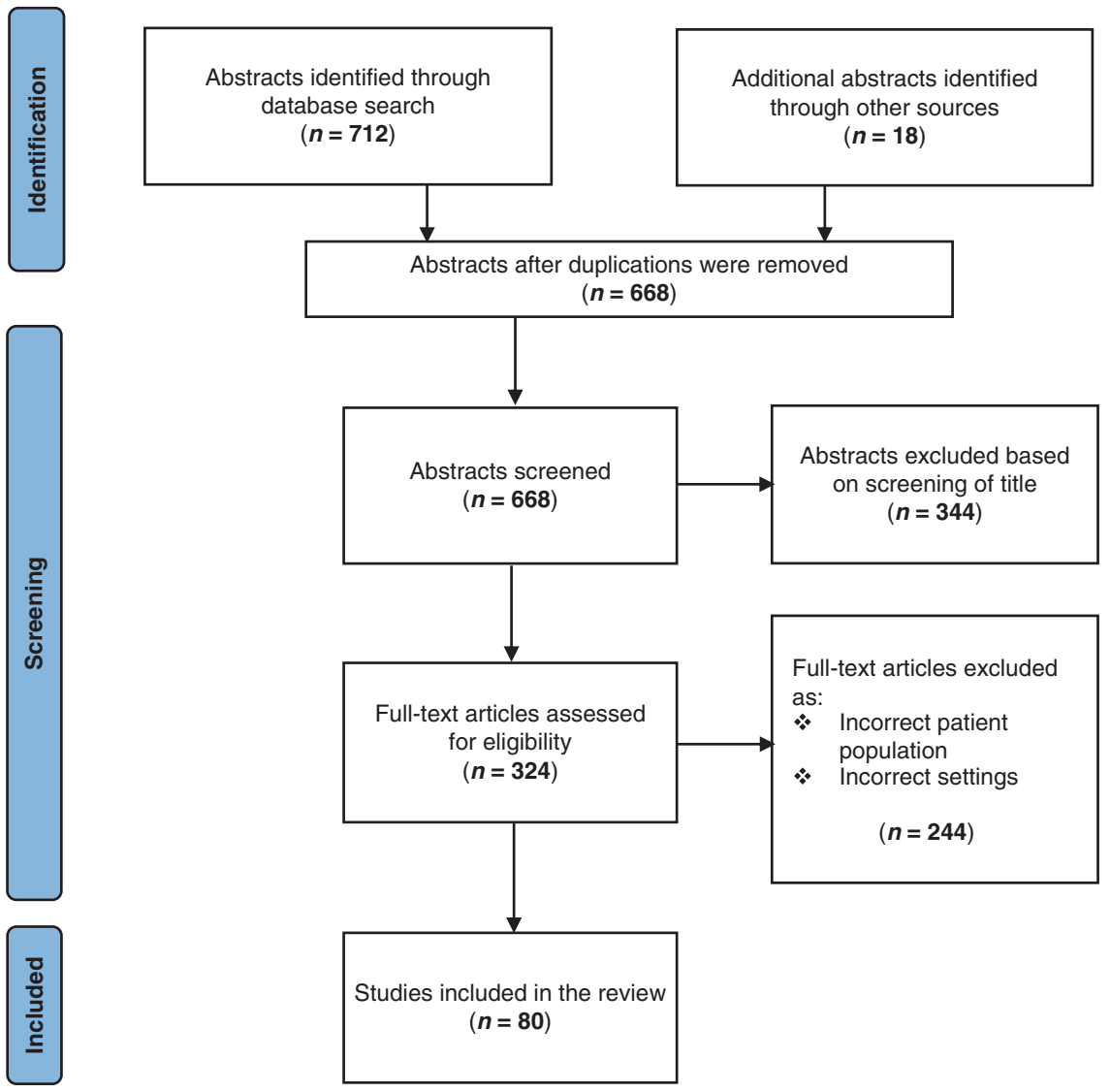

Fig. 1 PRISMA flow diagram of studies included in the systematic review of definitions of neonatal sepsis in randomised controlled trials.

Table 1. Definitions of neonatal sepsis by primary criteria.

\begin{tabular}{ll} 
Combination of primary criteria & N \\
Culture alone & 35 \\
\hline Culture + signs & 29 \\
\hline Signs + laboratory & 25 \\
Culture + signs + laboratory & 12 \\
\hline Signs alone & 7 \\
\hline Culture + labs & 6 \\
\hline Signs + radiology & 6 \\
\hline Laboratory alone & 4 \\
\hline Signs + risk factors & 2 \\
\hline Culture + laboratory + radiology & 1 \\
\hline Radiology alone & 1
\end{tabular}

examples given, or a prescriptive list), the remaining 40 definitions mentioned signs without specifying what signs were looked for 20 definitions explicitly stated how many signs had to be present, with a breakdown as follows: 1 sign $(n=8), 2$ signs $(n=11)$, and 3 signs $(n=1)$. To categorise the wide variety of clinical signs mentioned, signs were grouped by system, except for 'requirement for antibiotics', which was grouped alone.

Antibiotic requirement: Seven definitions mentioned a 'requirement for antibiotics' as a sign of neonatal sepsis. The date range for these seven definitions is 2000-2018. Six of the seven definitions mention a requirement for a 5-day course of antibiotics, intention to treat for 5 days, or neonatal death before completion of a 5-day antibiotic course.
Table 2. Definitions mentioning specific culture source.

\begin{tabular}{|c|c|}
\hline Culture source & $N$ \\
\hline Blood & 71 \\
\hline Cerebrospinal fluid & 29 \\
\hline Urine & 10 \\
\hline Skin/surface & 4 \\
\hline Pus [unspecified] & 3 \\
\hline Tracheal aspirate & 2 \\
\hline Synovial fluid & 1 \\
\hline Peritoneal fluid & 1 \\
\hline Intravascular catheter & 1 \\
\hline Any sterile site & 1 \\
\hline
\end{tabular}

Overview of clinical signs: The categories of clinical signs appeared in the following order of frequency: systemic $(n=$ $32)$, respiratory $(n=29)$, cardiovascular $(n=28)$, gastrointestinal signs $(n=12)$, neurological $(n=12)$, and miscellaneous $(n=8)$. The frequency of individual signs within each category is outlined in Table 3.

Systemic signs: The 32 definitions specifying constitutional signs represent information from 22 separate papers. The constitutional signs were further stratified into five main categories: lethargy, temperature, feeding, cry, and colour (Table 3).

Respiratory signs: The 29 definitions specifying respiratory signs represent information from 20 separate papers. The listed respiratory signs were further stratified into three main categories: hypoxaemic signs, signs of distress, and requirement for support (Table 3). 


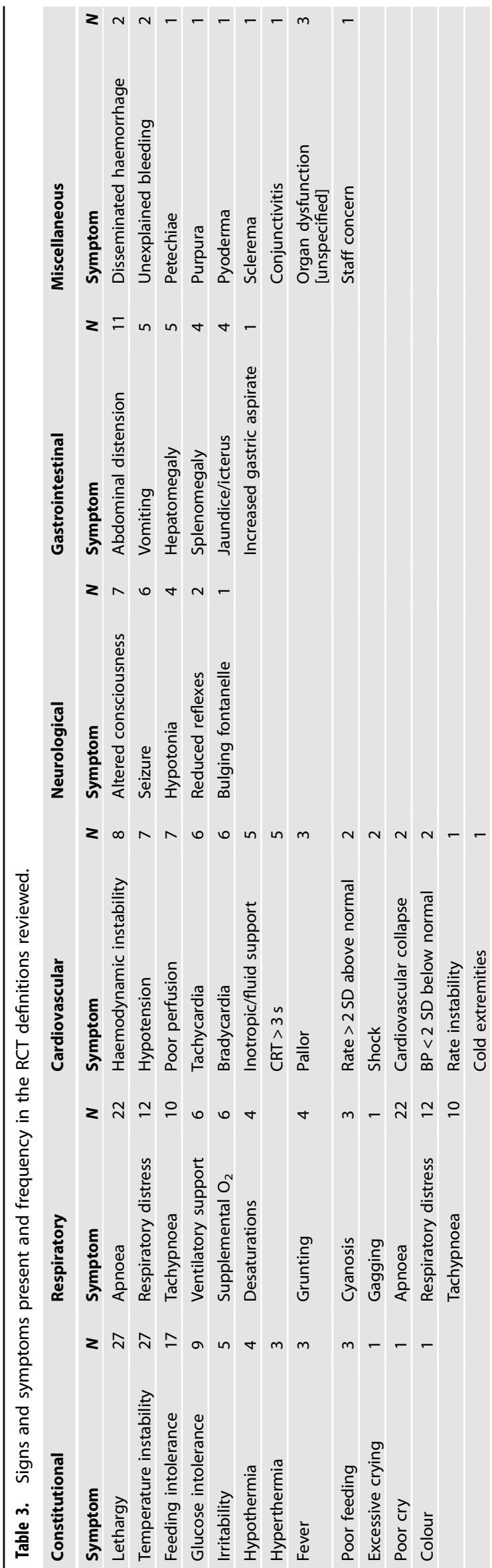

Cardiovascular signs: The 28 definitions specifying cardiovascular signs represents information from 19 separate papers. The listed cardiovascular signs were divided into three broad categories: heart rate, blood pressure, and indicators of perfusion (Table 3).

Neurological signs: The 12 definitions specifying neurological signs represent information from 10 individual papers. A total of five neurological signs were mentioned (Table 3).

Gastrointestinal signs: A total of six gastrointestinal signs were mentioned (Table 3).

Miscellaneous signs: The eight definitions that specified miscellaneous signs represent information from six individual papers. A total of nine miscellaneous signs were mentioned (Table 3).

(3) Laboratory (microbiological/haematological/biochemical) signs of infection

Haematological/biochemical signs of infection were a component of 48 definitions of neonatal sepsis, 4 of which defined sepsis based on laboratory criteria alone. The remaining 44 defined sepsis based on laboratory criteria in conjunction with other primary criteria (Table 1). The 48 laboratory-related definitions of sepsis came from 41 separate papers. Of the seven papers that provided two definitions, six papers included two definitions in order to distinguish between definite sepsis and probable sepsis. Of these six papers, three defined probable sepsis as laboratory criteria with clinical signs and definite sepsis as culture with laboratory criteria and clinical signs.

Although there were 48 laboratory-related definitions of sepsis, only 40 specified the laboratory tests required to make the diagnosis, while the remaining 8 mentioned laboratory parameters, without specifying what tests were necessary. There was a wide variation in the number of laboratory parameters included in each definition and of the 40 definitions that specified laboratory tests to be positive, 21 required at least one parameter to be abnormal, 18 requires at least 2 parameters to be abnormal, and 1 required at least 3 laboratory parameters to be abnormal. A total of 20 individual laboratory tests were mentioned by the 48 definitions of neonatal sepsis that included laboratory criteria as a primary criterion. These 20 individual parameters were grouped into five categories: blood count, serum inflammatory markers, metabolic markers, histopathological findings, and markers of pathogenaemia (Table 4).

(4) Radiological signs of infection

There were eight definitions of neonatal sepsis that included radiological signs of infection and only seven specified which radiological signs were sought. All seven definitions that specified signs specified chest X-ray/evidence of pneumonia as the only relevant radiological exam.

(5) Risk factors for infection

There were two definitions of neonatal sepsis that included risk factors for infection as a primary diagnostic criterion. The risk factors mentioned are as follows: amnionitis $(n=2)$, premature rupture of membranes $(n=2)$, foul-smelling amniotic fluid $(n=1)$, prolonged rupture of membranes $>24 \mathrm{~h}(n=1)$, maternal fever $(n=1)$, maternal urinary tract infection $(n=1)$.

\section{Qualifiers}

1. Early- versus late-onset neonatal sepsis

No definition of neonatal sepsis specified the timeframe for neonatal sepsis as other than birth to 28 days of life. Two studies provided definitions of both early- and late-onset sepsis. A further 11 studies provided definitions of either early or late-onset sepsis. Of the seven studies that defined 
Table 4. Frequency of laboratory signs in the RCT definitions reviewed.

\begin{tabular}{|c|c|}
\hline Laboratory signs & $N$ \\
\hline C-reactive protein & 30 \\
\hline Not specified & 9 \\
\hline$>5 \mathrm{mg} / \mathrm{L}$ & 1 \\
\hline$>9 \mathrm{mg} / \mathrm{L}$ & 1 \\
\hline$>10 \mathrm{mg} / \mathrm{L}$ & 13 \\
\hline$>12 \mathrm{mg} / \mathrm{L}$ & 1 \\
\hline$>20 \mathrm{mg} / \mathrm{L}$ & 4 \\
\hline$>60 \mathrm{mg} / \mathrm{L}$ & 1 \\
\hline White cell count (WCC) & 16 \\
\hline $\mathrm{I}: \mathrm{T}$ ratio & 15 \\
\hline Neutrophil count & 13 \\
\hline Platelet count & 10 \\
\hline Micro-ESR & 8 \\
\hline Band cell count & 7 \\
\hline Full blood count (FBC) & 3 \\
\hline IL-6 & 3 \\
\hline Glucose & 3 \\
\hline Toxic granules in peripheral smear & 2 \\
\hline Bacterial antigen & 2 \\
\hline TNF-alpha & 1 \\
\hline Procalcitonin & 1 \\
\hline Lactate & 1 \\
\hline $\mathrm{pH}$ & 1 \\
\hline Histologic diagnosis of pneumonia & 1 \\
\hline Cerebrospinal WCC & 1 \\
\hline Viral polymerase chain reaction (PCR) & 1 \\
\hline CSF Gram stain & 1 \\
\hline
\end{tabular}

early-onset sepsis, one study defined early onset as arising < $48 \mathrm{~h}$ of life, five studies as $<72 \mathrm{~h}$, and one study as $<5$ days of life. Of the eight studies that defined late-onset sepsis, all eight defined late-onset sepsis as arising $\geq 72 \mathrm{~h}$ of life.

2. Definite/probable/possible sepsis

There were 21 studies that provided definitions that discriminated between degrees of certainty of the diagnosis of sepsis. Of utmost certainty were 'definite sepsis' or 'culture-proven sepsis', next was 'probable sepsis' or 'clinical sepsis', followed by 'possible sepsis' as defined by some studies.

3. Stringency for coagulase-negative Staphylococci (CoNS)

Six papers included criteria relating to CONS. Of these, two specified that Staph. epidermidis was disregarded if cultured, and the other four papers (three mention CONS, one mentions Staph. epi.) specified additional criteria required to make the diagnosis if the culture yielded CONS/Staph. epi. Of these additional criteria, two papers required concomitant abnormalities of the white blood cell count or CRP, one paper required positive cultures from two separate sites (usually drawn simultaneously), and one required culture to be positive within $48 \mathrm{~h}$.

4. Septic shock/severe sepsis

Two papers specified additional criteria defining either septic shock or severe sepsis. The paper defining septic shock specified it as meeting the criteria for sepsis, plus blood pressure below the fifth centile for age requiring fluid resuscitation or inotropic support. The paper defining severe sepsis specified it as meeting the criteria for sepsis plus objective evidence of organ dysfunction.

\section{DISCUSSION}

There is considerable heterogeneity in the definitions of neonatal sepsis, both in the combinations of primary criteria used to define sepsis and in the specific secondary criteria in each category. This is huge problem for neonatology resulting in studies with most definitions not validated, sensitive or specific and using subjective criteria so not comparable or generalisable. Most notably, there was a reliance on microbiological culture for definitive diagnosis of sepsis in $85 \%$ of studies. This suggests that most of these definitions actually aim to describe 'bacteraemia' rather than 'sepsis'. This is at variance with the adult Sepsis-3 guidelines ${ }^{9,34}$ and in contrast with the recent definitions of adult and paediatric sepsis which involve an 'inflammatory host responses with end organ impairment' independent of microbial detection. In additon, clinical signs of neonatal sepsis and infection are notoriously unreliable for diagnosis and can only trigger evaluation and may not be part of an actual definition as they are not specific for sepsis in neonates.

Most definitions using microbiological culture as an essential component for the diagnosis of sepsis vary in the exact requisites for a positive culture. Most papers specified a culture site and of those that did, most specified blood as the only site while a minority included other possible sites (e.g. CSF). Of the 83 definitions that included culture as a criterion, only 12 outlined what pathogens were assumed to be pathogenic which is significant in the context of CONS and the possibility of culture contamination versus nosocomial infection. Commensals represent a diagnostic quandary for neonatal sepsis, and approaches to CoNS detected in culture varied widely. No paper mentioned decisions in cases of a polymicrobial culture. $^{115}$

Most definitions included clinical signs as a component of the definition of sepsis; however, papers varied in the precise definitions of certain signs and the overall list of signs. Certain signs predominate in frequency (Table 3 ) such as systemic signs, including lethargy, feeding intolerance and temperature instability, and cardiorespiratory signs including hypotension and respiratory distress. Two of the most frequent signs listedhypotension and respiratory distress-as well as the platelet count are parameters of organ failure measured in the SOFA score in the Sepsis- 3 guidelines $^{9}$ and are identical to the three used for nSOFA score. ${ }^{116}$

Severe sepsis is now widely regarded to be an obsolete characterisation of the syndrome; however, it is notable that the study that defined severe sepsis characterised it as the presence of organ dysfunction, which is the key feature of the Sepsis-3 definition of sepsis in adults. Septic shock is a useful clinical distinction in adults and neonates, but one definition specifically labels septic shock and other definitions include instead low blood pressure in the definition of sepsis. ${ }^{9}$

A wide range of laboratory investigations appeared in the results, 36 definitions mentioned abnormalities of the white cell count-or related measures like the neutrophil count, immature neutrophil count, and immature:total neutrophil ratio and inflammation as marked by CRP or ESR. While radiological signs of infection were classed as a primary criterion, this in effect meant signs of pneumonia on chest X-ray, given that this was the only radiological investigation cited by any of the studies. Risk factors for infection were cited by several studies, which included a comprehensive range of risk factors such as chorioamnionitis, prolonged rupture of membranes, or foul-smelling amniotic fluid. There was variability in the timeframes defined for early-onset neonatal sepsis $(<2,<3,<5$ days) with better agreement on the definition of late-onset sepsis. 
Definitions of neonatal sepsis used in randomised clinical trials demonstrate considerable variability which means that comparison of studies and outcomes is very challenging. ${ }^{117}$ The major neonatal organisations such as National Institute of Child Health and Human Development (NICHD) Neonatal Research Network, Centers for Disease Control and Prevention (CDC), Nosocomial infection surveillance system for preterm infants on neonatology departments (NEO-KISS), Vermont Oxford Network, and the Canadian Neonatal Network also differ considerably in defining early and late-onset sepsis, the requirement for antibiotic use or clinical signs, and in the rules for including CoNS as pathogens. ${ }^{34}$

A concentration on microbiological culture persists in contrast to definitions used for children and adults with less inclusion of multiorgan dysfunction. The next steps in this process include a Delphi process including stakeholders internationally to develop a consensus definition with regular updates. ${ }^{118,119}$ The involvement of families is crucial in this process to ensure a definition that can be translated across healthcare professionals and families. ${ }^{119,120}$ Definitions of neonatal sepsis require standardisation for accurate diagnoses, epidemiological studies and data synthesis. Any sepsis definition that includes organ dysfunction first requires definition of normal organ function in the vulnerable preterm population which is still enormous challenge for the neonatologists internationally. Additional collection and analysis of clinical data of the physiology of the extreme preterm is necessary to define hypotension, thrombocytopenia, renal and liver dysfunction before including 'end organ impairment' as a critical factor for defining neonatal sepsis.

\section{REFERENCES}

1. Fleischmann-Struzek, C. et al. The global burden of paediatric and neonatal sepsis: a systematic review. Lancet Resp. Med. 6, 223-230 (2018).

2. United Nations Inter-agency Group for Child Mortality Estimation (UN IGME). Levels \& Trends in Child Mortality: Report 2019, Estimates developed by the United Nations Inter-agency Group for Child Mortality Estimation (United Nations Children's Fund, New York, 2019).

3. Iroh Tam, P. Y. \& Bendel, C. M. Diagnostics for neonatal sepsis: current approaches and future directions. Pediatr. Res. 82, 574-583 (2017).

4. Cantey, J. B. \& Sánchez, P. J. J. Pediatr. 159, 707-770 (2011).

5. Cantey J. B. \& Baird S. D. Pediatrics 140, e20170044 (2017).

6. The INIS Collaborative Group. Treatment of neonatal sepsis with intravenous immune globulin. N. Engl. J. Med. 365, 1201-1212 (2011).

7. The ELFIN Trial Investigators Group. Enteral lactoferrin supplementation for very preterm infants: a randomised placebo-controlled trial. Lancet 393, 423-434 (2019).

8. Geneva World Health Organisation. Global report of the epidemiology and burden of sepsis, current evidence, identifying gaps and future directions. (2020).

9. Singer, M. et al. The Third International Consensus Definitions for Sepsis and Septic Shock (Sepsis-3). JAMA 315, 801-810 (2016).

10. Wynn, J. \& Polin, R. A. A Neonatal Sequential Organ Failure Assessment Score predicts mortality to late-onset sepsis in preterm very low birth weight infants. Pediatr. Res 88, 85-90 (2020).

11. Klinger et al. Outcome of early-onset sepsis in a national cohort of very low birth weight infants. Pediatrics 125, e736-e740 (2010).

12. Stoll, B. J. et al. Late-onset sepsis in very low birth weight neonates: the experience of the NICHD Neonatal Research Network. Pediatrics 110, 285-291 (2002).

13. Stoll, B. J. et al. Neurodevelopmental and growth impairment among extremely low-birth-weight infants with neonatal infection. JAMA 292, 2357-2365 (2004).

14. Van der Ree et al. Functional impairments at school age of preterm born children with late-onset sepsis. Early Hum. Dev. 87, 821-826 (2011).

15. Luregn, J. et al. Impact of sepsis on neurodevelopmental outcome in a Swiss National Cohort of extremely premature infants. Pediatrics 128, e348-e357 (2011).

16. Hannah, C. et al. Recurrent postnatal infections are associated with progressive white matter injury in premature infants. Pediatrics 122, 299-305 (2008).

17. Mitha, A. et al. Neonatal infection and 5-year neurodevelopmental outcome of very preterm infants. Pediatrics 132, e372 (2013).
18. Dammann, O., Kuban, K. C. K. \& Leviton, A. Perinatal infection, fetal inflammatory response, white matter damage, and cognitive limitations in children born preterm. Ment. Retard Dev. Disabil. Res Rev. 8, 46-50 (2008).

19. Kuppala, V. S., Meinzen-Derr, J., Morrow, A. L. \& Schibler, K. R. Prolonged initial empirical antibiotic treatment is associated with adverse outcomes in premature infants. J. Paediatr. 159, 720-725 (2011).

20. Ting, J. Y. et al., Canadian Neonatal Network Investigators. Association between antibiotic use and neonatal mortality and morbidities in very low-birthweight infants without culture-proven sepsis or necrotizing enterocolitis. JAMA Pediatr. 170, 1181-1187 (2016).

21. Cotton, C. M. et al., NICHD Neonatal Research Network. Prolonged duration of initial empirical antibiotic treatment is associated with increased rates of necrotizing enterocolitis and death for extremely low birth weight infants. Pediatrics 123, 58-66 (2009).

22. Wickens, K., Pearce, N., Crane, J. \& Beasley, R. Antibiotic use in early childhood and the development of asthma. Clin. Exp. Allergy 29, 766-771 (1999).

23. Johnson, C. C. et al. Antibiotic exposure in early infancy and risk for childhood atopy. J. Allergy Clin. Immunol. 115, 1218-1224 (2005).

24. Fouhy, F. et al. High-throughput sequencing reveals the incomplete, short-term recovery of infant gut microbiota following parenteral antibiotic treatment with ampicillin and gentamicin. Antimicrob. Agents Chemother. 56, 5811-5820 (2012).

25. Tanaka, S. et al. Influence of antibiotic exposure in the early postnatal period on the development of intestinal microbiota. FEMS Immunol. Med. Microbiol. 56, 80-87 (2009).

26. Torrazza, R. \& Neu, J. The developing intestinal microbiome and its relationship to health and disease in the neonate. J. Perinatol. 31, S29-S34 (2011).

27. Heijtz, R. D. Fetal, neonatal, and infant microbiome: perturbations and subsequent effects on brain development and behaviour. Semin. Fetal Neonatal Med. 21, 410-417 (2016)

28. Douglas-Escobar, M., Elliott, E. \& Neu, J. Effect of intestinal microbial ecology on the developing brain. JAMA Pediatr. 167, 374-379 (2013).

29. Leroux, S., Zhao, W., Bétrémieux $P$ on behalf of the French Society of Neonatology, et al. Therapeutic guidelines for prescribing antibiotics in neonates should be evidence-based: a French national survey. Arch. Dis. Child 100, 394-398 (2015).

30. Cotton, C. M. Antibiotic stewardship: reassessment of guidelines for management of neonatal sepsis. Clin. Perinatol. 42, 195-206 (2015).

31. Engle, W. D. et al. Neonatal pneumonia: comparison of 4 vs 7 days of antibiotic therapy in term and near-term infants. J. Perinatol. 20, 421-426 (2000).

32. Wójkowska-Mach, J. et al. Necrotising enterocolitis in preterm infants: epidemiology and antibiotic consumption in the Polish Neonatology Network neonatal intensive careunits in 2009. PLoS ONE 9, e92865 (2014).

33. Costeloe, K. et al. Sharing data to accelerate medicine development and improve neonatal care: data standards and harmonized definitions. J. Pediatr. 203, 437-441 (2018).

34. McGovern, M. et al. Challenges in developing a consensus definition of neonatal sepsis. Pediatr. Res. 88, 14-26 (2020).

35. Saeidi et al. Clinical and biochemical effects of recombinant human granulocyte colony-stimulating factor on the prognosis of preterm infants with early onset neonatal sepsis. Arch. Pediatr. Infect. Dis. 7, e67807 (2019).

36. Edwards et al. The effect of prophylactic ointment therapy on nosocomial sepsis rates and skin integrity in infants with birth weights of 501 to $1000 \mathrm{~g}$. Pediatrics 113, 1195-1203 (2004)

37. Morris et al. Immediate delivery compared with expectant management after preterm pre-labour rupture of the membranes close to term (PPROMT trial): a randomised controlled trial. Lancet 387, 444-452 (2016).

38. Haque, K. N. "Pentaglobin" in the treatment of neonatal sepsis. J. Obstet. Gynaecol. 10, S25-S26 (1989).

39. Van der, Ham. et al. Induction of labor versus expectant management in women with preterm prelabor rupture of membranes between 34 and 37 weeks: a randomized controlled trial. PLoS Med. 9, e1001208 (2012).

40. Mercer et al. The antibiotic treatment of PPROM study: systemic maternal and fetal markers and perinatal outcomes. Am. J. Obstet. Gynecol. 206, 145.e1-145.e9 (2012).

41. Zhang et al. Timing of antibiotic prophylaxis in elective caesarean delivery: a multi-center randomized controlled trial and meta-analysis. PLOS ONE 10, e0129434 (2015).

42. Kaur et al. Efficacy of bovine lactoferrin supplementation in preventing lateonset sepsis in low birth weight neonates: a randomized placebo-controlled clinical trial. J. Trop. Pediatr. 61, 370-376 (2015).

43. Naef et al. Premature rupture of membranes at 34 to 37 weeks' gestation: aggressive versus conservative management. Am. J. Obstet. Gynecol. 178, 126-130 (1998). 
44. Violaris et al. Comparison of fluconazole and nystatin oral suspensions for prophylaxis of systemic fungal infection in very low birthweight infants. Am. J. Perinatol. 27, 73-78 (2010).

45. Rohatgi et al. Seven versus 10 days antibiotic therapy for culture-proven neonatal sepsis: a randomised controlled trial. J. Paediatr. Child Health 53, 556-562 (2017).

46. Mehta et al. Oral zinc supplementation for reducing mortality in probable neonatal sepsis: a double blind randomized placebo controlled trial. Indian Pediatr. 50, 390-393 (2013).

47. Roos et al. Effect of maintenance tocolysis with nifedipine in threatened preterm labor on perinatal outcomes: a randomized controlled trial. JAMA 309, 41-47 (2013).

48. Pattinson et al. The use of dexamethasone in women with preterm premature rupture of membranes-a multicentre, double-blind, placebo-controlled, randomised trial. South Afr. Med. J. 89, 865-870 (1999).

49. Bordbar et al. Standard multiple and single daily dosing of amikacin in premature infants. Iran. J. Neonatol. 8, 57-64 (2017).

50. Roca et al. Oral azithromycin given during labour decreases bacterial carriage in the mothers and their offspring: a double-blind randomized trial. Clin. Microbiol. Infect. 22, 565 (2016).

51. Cutland, C. L. et al. Chlorhexidine maternal-vaginal and neonate body wipes in sepsis and vertical transmission of pathogenic bacteria in South Africa: a randomised, controlled trial. Lancet 374, 1909-1916 (2009).

52. Karagol et al. Randomized controlled trial of slow vs rapid enteral feeding advancements on the clinical outcomes of preterm infants with birth weight 750-1250 g. J. Parenter. Enter. Nutr. 37, 223-228 (2013).

53. Stocker et al. Procalcitonin-guided decision making for duration of antibiotic therapy in neonates with suspected early-onset sepsis: a multicentre, randomised controlled trial (NeoPIns). Lancet 390, 871-881 (2017).

54. Hikaru et al. Bifidobacteria prevents preterm infants from developing infection and sepsis. Int J. Probiotics Prebiotics 5, 33-36 (2010).

55. Rouse et al. Chlorhexidine vaginal irrigation for the prevention of peripartal infection: a placebo-controlled randomized clinical trial. Am. J. Obstet. Gynecol. 176, 617-622 (1997).

56. Strus et al. Effects of oral probiotic supplementation on gut Lactobacillus and Bifidobacterium populations and the clinical status of low-birth-weight preterm neonates: a multicenter randomized, double-blind, placebo-controlled trial. Infect. Drug Resist. 11, 1557-1571 (2018).

57. Van der Hoogen et al. In-line filters in central venous catheters in a neonatal intensive care unit. J. Perinat. Med. 34, 71-74 (2006).

58. Calkins et al. Low-dose parenteral soybean oil for the prevention of parenteral nutrition-associated liver disease in neonates with gastrointestinal disorders. J. Parenter. Enteral Nutr. 41, 404-411 (2017).

59. Manzoni et al. Bovine lactoferrin supplementation for prevention of late-onset sepsis in very low-birth-weight neonates: a randomized trial. JAMA 302, 1421-1428 (2009).

60. Shabaan, et al.Conventional versus prolonged infusion of meropenem in neonates with Gram-negative late-onset sepsis: a randomized controlled trial. Pediatr. Infect. Dis. J. 36, 358-363 (2017).

61. Romeo et al. Role of probiotics in the prevention of the enteric colonization by Candida in preterm newborns: incidence of late-onset sepsis and neurological outcome. J. Perinatol. 31, 63-69 (2011)

62. Thompson et al. A randomized, controlled trial of parenteral glutamine in ill, very low birth-weight neonates. J. Pediatr. Gastroenterol. Nutr. 37, 550-554 (2003).

63. Lewis et al. Preterm premature ruptured membranes: a randomized trial of steroids after treatment with antibiotics. Obstet. Gynecol. 88, 801-805 (1996).

64. Baske et al. Epinephrine versus dopamine in neonatal septic shock: a doubleblind randomized controlled trial. Eur. J. Pediatr. 177, 1335-1342 (2018).

65. Weisman et al. Intravenous immune globulin prophylaxis of late-onset sepsis in premature neonates. J. Pediatr. 125, 922-930 (1994).

66. Ochoa et al. Randomized controlled trial of lactoferrin for prevention of sepsis in Peruvian neonates less than 2500 g. Pediatr. Infect. Dis. J. 34, 571-576 (2015).

67. Ikram et al. A randomised controlled trial of glutamine-enriched neonatal parenteral nutrition in Malaysia. Singapore Med. J. 52, 356-360 (2011).

68. Kashanian et al. Comparison between two doses of betamethasone administration with $12 \mathrm{~h}$ vs. $24 \mathrm{~h}$ intervals on prevention of respiratory distress syndrome: a randomised trial. J. Obstet. Gynaecol. 38, 770-776 (2018).

69. Sanghvi et al. Feasibility of exclusive enteral feeds from birth in VLBW infants $>1200$ g-an RCT. Acta Paediatr. Int. J. Paediatr. 102, 299-304 (2013).

70. Thigpen et al. Timing of prophylactic antibiotic administration in the uninfected laboring gravida: a randomized clinical trial. Am. J. Obstet. Gynecol. 192, 1864-1871 (2005)
71. Miura et al. A randomized, double-masked, placebo-controlled trial of recombinant granulocyte colony-stimulating factor administration to preterm infants with the clinical diagnosis of early-onset sepsis. Pediatrics 107, 30-35 (2001).

72. Balachandran et al. Bovine colostrum in prevention of necrotizing enterocolitis and sepsis in very low birth weight neonates: a randomized, double-blind, placebo-controlled pilot trial. J. Trop. Pediatr. 63, 10-17 (2017).

73. Aggarwal et al. Selenium supplementation for prevention of late-onset sepsis in very low birth weight preterm neonates. J. Trop. Pediatr. 62, 185-193 (2016).

74. Morris et al. Immediate delivery versus expectant care in women with preterm prelabour rupture of the membranes close to term (PPROMT): a multi-centre randomised controlled trial. Arch. Dis. Child 99, A223 (2014)

75. Odio et al. Comparative efficacy of ceftazidime vs. carbenicillin and amikacin for treatment of neonatal septicemia. Pediatr. Infect. Dis. J. 6, 371-377 (1987).

76. Newton et al. Effect of zinc supplementation on early outcome of neonatal sepsis-a randomized controlled trial. Indian J. Pediatr. 83, 289-293 (2016).

77. Van Der Ham et al. Management of late-preterm premature rupture of membranes: the PPROMEXIL-2 trial. Am. J. Obstet. Gynecol. 207, 276 (2012).

78. Haque et al. IgM-enriched intravenous immunoglobulin therapy in neonatal sepsis. Am. J. Dis. Child 142, 1293-1296 (1988).

79. Haque et al. Intravenous immunoglobulin for prevention of sepsis in preterm and low birth weight infants. Pediatr. Infect. Dis. 5, 622-625 (1986).

80. Cararach et al. Administration of antibiotics to patients with rupture of membranes at term: a prospective, randomized, multicentric study. Collaborative Group on PROM. Acta Obstet. Gynecol. Scand. 77, 298-302 (1998).

81. Regan et al. Colonization with group B streptococci in pregnancy and adverse outcome. Am. J. Obstet. Gynecol. 174, 1354-1360 (1996).

82. Kalaranjini et al. Comparison of administration of single dose ceftriaxone for elective caesarean section before skin incision and after cord clamping in preventing post-operative infectious morbidity. Arch. Gynecol. Obstet. 288 1263-1268 (2013).

83. Egerman et al. A randomized, controlled trial of oral and intramuscular dexamethasone in the prevention of neonatal respiratory distress syndrome. Am. J. Obstet. Gynecol. 179, 1120-1123 (1998).

84. Eriksen et al. Chlorhexidine vs. sterile vaginal wash during labor to prevent neonatal infection. Infect. Dis. Obstet. Gynecol. 5, 286-290 (1997).

85. Yaramis et al. A randomized trial of granulocyte-macrophage colony-stimulating factor in neonates with sepsis and neutropenia. Pediatrics 107, 36-41 (2001).

86. Chowdhary et al. Randomized controlled trial of 7-day vs. 14-day antibiotics for neonatal sepsis. J. Trop. Pediatr. 52, 427-432 (2006).

87. Sullivan et al. Administration of cefazolin prior to skin incision is superior to cefazolin at cord clamping in preventing postcesarean infectious morbidity: a randomized, controlled trial. Am. J. Obstet. Gynecol. 196, 455 (2007).

88. Lauterbach et al. Pentoxifylline reduces plasma tumour necrosis factor-alpha concentration in premature infants with sepsis. Eur. J. Pediatr. 155, 404-409 (1996).

89. Drossou, V. et al. In vivo effect of rhG-CSF on the number and function of neonatal neutrophils during sepsis. A randomized controlled trial. Pediatr. Res 56, 752 (1997).

90. Banupriya et al. Short term oral zinc supplementation among babies with neonatal sepsis for reducing mortality and improving outcome-a double-blind randomized controlled trial. Indian J. Pediatr. 85, 5-9 (2018).

91. Nabhan et al. Antibiotic prophylaxis in prelabor spontaneous rupture of fetal membranes at or beyond 36 weeks of pregnancy. Int J. Gynecol. Obstet. 124, 59-62 (2014).

92. Hemels et al. Prevention of neonatal late-onset sepsis associated with the removal of percutaneously inserted central venous catheters in preterm infants. Pediatr. Crit. Care Med. 12, 445-448 (2011).

93. Aradhya et al. Double Volume Exchange Transfusion in Severe Neonatal Sepsis. Indian J. Pediatr. 83, 107-113 (2016).

94. Akdag et al. Role of pentoxifylline and/or IgM-enriched intravenous immunoglobulin in the management of neonatal sepsis. Am. J. Perinatol. 31, 905-912 (2014).

95. Gupta et al. Evaluation of efficacy of skin cleansing with chlorhexidine in prevention of neonatal nosocomial sepsis - a randomized controlled trial. J. Matern neonatal Med. 29, 242-247 (2016).

96. Birch et al. A randomised, controlled trial of heparin in total parenteral nutrition to prevent sepsis associated with neonatal long lines: The Heparin in Long Line Total Parenteral Nutrition (HILLTOP) trial. Arch. Dis. Child Fetal Neonatal Ed. 95, 252-257 (2010).

97. Sorokin et al. Maternal serum interleukin-6, C-reactive protein, and matrix metalloproteinase- 9 concentrations as risk factors for preterm birth $<32$ weeks and adverse neonatal outcomes. Am. J. Perinatol. 27, 631-640 (2010).

98. Berghella et al. Prevention of preterm birth with a pessary in twins (PoPPT): a randomized controlled trial. Am. J. Obstet. Gynecol. 216, 28 (2017) 
99. Mendes et al. Massage therapy reduces hospital stay and occurrence of lateonset sepsis in very preterm neonates. J. Perinatol. 28, 815-820 (2008).

100. Bajcetic et al. Antioxidative system in the erythrocytes of preterm neonates with sepsis: The effects of vitamin E supplementation. Ann. Clin. Biochem. 51, 550-556 (2014).

101. Maamouri et al. The impact of oral glutamine supplementation on prevention of nosocomial infections in preterm infants. Iran. J. Neonatol. 7, 19-24 (2016).

102. Tagare et al. Routine antibiotic use in preterm neonates: a randomised controlled trial. J. Hosp. Infect. 74, 332-336 (2010).

103. Ceriani et al. Clinical outcome of neonates with nosocomial suspected sepsis treated with cefazolin or vancomycin. A non-inferiority, randomized, controlled trial. Arch. Argent. Pediatr. 112, 308-314 (2014).

104. Driessen et al. Fluconazole versus amphotericin B for the treatment of neonatal fungal septicemia: a prospective randomized trial. Pediatr. Infect. Dis. J. 15, 1107-1112 (1996).

105. Gathwala et al. Effect of topical application of chlorhexidine for umbilical cord care in comparison with conventional dry cord care on the risk of neonatal sepsis: a randomized controlled trial. J. Trop. Pediatr. 59, 209-213 (2013).

106. Abzug et al. A randomized, double-blind, placebo-controlled trial of pleconaril for the treatment of neonates with enterovirus sepsis. J. Pediatr. Infect. Dis. Soc. 5, 53-62 (2016).

107. Erdemir et al. The effect of topical ointment on neonatal sepsis in preterm infants. J. Matern. Neonatal Med. 28, 33-36 (2015).

108. Saini et al. Short course versus 7-day course of intravenous antibiotics for probable neonatal septicemia: a pilot, open-label, randomized controlled trial. Indian Pediatr. 48, 19-24 (2011).

109. Shenoi et al. Multicenter randomized placebo controlled trial of therapy with intravenous immunoglobulin in decreasing mortality due to neonatal sepsis. Indian Pediatr. 36, 1113-1118 (1999).

110. Miura et al. Assessing the efficacy of the recombinant human granulocyte colony- stimulating factor "rhG-CSF" in the treatment of early neonatal sepsis in premature neonates. J. Pediatr. 76, 193-199 (2000).

111. El-Ganzoury et al. In vivo effect of recombinant human granulocyte colony-stimulating factor on neutrophilic expression of CD11b in septic neonates: a randomized controlled trial. Pediatr. Hematol. Oncol. 29, 272-284 (2012).

112. Goel et al. Prophylactic antibiotics and sepsis in neonates born through meconium stained amniotic fluid (MSAF)-a randomized controlled trial. Arch. Dis. Child 97, A115 (2012).

113. Alcock et al. Prevention of neonatal late-onset sepsis: a randomised controlled trial. BMC Pediatr. 2017;17

114. Banupriya et al. Efficacy of zinc supplementation on serum calprotectin, inflammatory cytokines and outcome in neonatal sepsis - a randomized controlled trial. J. Matern Neonatal Med. 30, 1627-1631 (2017).

115. Molloy, E. J. \& Strunk, T. Role of C-reactive protein for late-onset neonatal sepsis. JAMA Pediatr. 175, 100-101 (2021).

116. Fleiss, N. et al. Evaluation of the neonatal sequential organ failure assessment and mortality risk in preterm infants with late-onset infection. JAMA Netw. Open 4, e2036518 (2021).

117. Molloy, E. J. et al. Neonatal sepsis: need for consensus definition, collaboration and core outcomes. Pediatr. Res. 88, 2-4 (2020).
118. Allen, J., Brenner, M., Hauer, J., Molloy, E. \& McDonald, D. Severe Neurological Impairment: a delphi consensus-based definition. Eur. J. Paediatr. Neurol. 29, 81-86 (2020).

119. Allen, J., Molloy, E. \& McDonald, D. Severe neurological impairment: a review of the definition. Dev. Med Child Neurol. 62(Mar), 277-282 (2020).

120. Molloy, E. J., Mader, S., Modi, N., \& Gale, C. Parent, child and public involvement in child health research: core value not just an optional extra. Pediatr. Res. 85, 2-3 (2019).

121. Carr, R., Brocklehurst, P., Doré, C. J. \& Modi, N. Granulocyte-macrophage colony stimulating factor administered as prophylaxis for reduction of sepsis in extremely preterm, small for gestational age neonates (the PROGRAMS trial): a singleblind, multicentre, randomised controlled trial. Lancet. 373, 226-233 (2009).

122. Goel, A. et al. Role of prophylactic antibiotics in neonates born through meconiumstained amniotic fluid (MSAF) - a randomized controlled trial. Eur J Pediatr. 174, 237-243 (2014).

\section{ACKNOWLEDGEMENTS}

Series was funded by ESPR.

\section{COMPETING INTERESTS}

The authors declare no competing interests.

\section{ADDITIONAL INFORMATION}

Correspondence and requests for materials should be addressed to Eleanor J. Molloy.

Reprints and permission information is available at http://www.nature.com/ reprints

Publisher's note Springer Nature remains neutral with regard to jurisdictional claims in published maps and institutional affiliations.

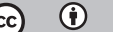

Open Access This article is licensed under a Creative Commons Attribution 4.0 International License, which permits use, sharing, adaptation, distribution and reproduction in any medium or format, as long as you give appropriate credit to the original author(s) and the source, provide a link to the Creative Commons license, and indicate if changes were made. The images or other third party material in this article are included in the article's Creative Commons license, unless indicated otherwise in a credit line to the material. If material is not included in the article's Creative Commons license and your intended use is not permitted by statutory regulation or exceeds the permitted use, you will need to obtain permission directly from the copyright holder. To view a copy of this license, visit http://creativecommons. org/licenses/by/4.0/.

(c) The Author(s) 2021 